\title{
Bifurcation of an Orbit Homoclinic to a Hyperbolic Saddle of a Vector Field in $\mathbb{R}^{4}$
}

\author{
Tiansi Zhang and Dianli Zhao \\ College of Science, University of Shanghai for Science and Technology, Shanghai 200093, China \\ Correspondence should be addressed to Tiansi Zhang; zhangts1209@163.com
}

Received 13 August 2014; Accepted 8 September 2014

Academic Editor: Li Li

Copyright (C) 2015 T. Zhang and D. Zhao. This is an open access article distributed under the Creative Commons Attribution License, which permits unrestricted use, distribution, and reproduction in any medium, provided the original work is properly cited.

We perform a bifurcation analysis of an orbit homoclinic to a hyperbolic saddle of a vector field in $\mathbb{R}^{4}$. We give an expression of the gap between returning points in a transverse section by renormalizing system, through which we find the existence of homoclinicdoubling bifurcation in the case $1+\alpha>\beta>v$. Meanwhile, after reparametrizing the parameter, a periodic-doubling bifurcation appears and may be close to a saddle-node bifurcation, if the parameter is varied. These scenarios correspond to the occurrence of chaos. Based on our analysis, bifurcation diagrams of these bifurcations are depicted.

\section{Introduction and Problems}

Homoclinic orbits are crucial to know dynamics of differential systems in many application fields. For example, the famous FitzHugh-Nagumo equations, given by PDEs (see [1]), describe how action potentials in neurons occur and spread

$$
\begin{gathered}
u_{t}=u_{x} x-f_{a}(u)-w, \\
w_{t}=\varepsilon(u-\gamma w),
\end{gathered}
$$

where $f_{a}(u)=u(u-a)(u-1)$. Through the variable transforming $\zeta=x+c t$, system (1) is then in an ODE form:

$$
\begin{gathered}
\dot{u}=v, \\
\dot{v}=c v+f_{a}(u)+w, \\
\dot{w}=\frac{\varepsilon}{c}(u-\gamma w) .
\end{gathered}
$$

It has an orbit homoclinic to the equilibrium $(u, v, w)=0$ which corresponds to a solitary wave $(u, w)(x, t)=(u, w)(\zeta)$ of system (1). The authors detected how homoclinic branch converted a 1-homoclinic orbit to a $\mathrm{N}$-homoclinic orbit.

In [2], a reversible water wave model was studied:

$$
\frac{2}{15} v^{i v}-b v^{\prime \prime}+a v+\frac{3}{2} v^{2}-\frac{1}{2}\left(v^{\prime}\right)^{2}+\left[v v^{\prime}\right]^{\prime}=0 .
$$

The system admits a flip orbit

$$
r(t)=3\left(b+\frac{1}{2}\right) \operatorname{sech}^{2}\left(t \sqrt{\frac{3}{4}(2 b+1)}\right)
$$

for $b>2, a>0$, and shows the existence of the N-homoclinic orbit in some circumstances on two sides of the flip bifurcation.

In fact, the homoclinic-doubling bifurcation, which switches a $2^{n-1}$-homoclinic orbit to a $2^{n}$-homoclinic orbit, exists extensively in systems with flips; see [3-6] and the references therein. A simple and analytic model permitting these flips was initially given by Sandstede in a threedimensional system in [7]. From then on, more and more excellent work has been done based on the model (see, e.g., $[8,9])$. Now researchers even extend these flips phenomena to heterodimensional cycles and homoclinic bellows to study periodic orbits and homoclinic orbits; see [10-12]. But none of them aimed to investigate the homoclinic-doubling bifurcations. So in this paper we focus on the homoclinic-doubling problem for a kind of homoclinic flips.

Throughout the paper, we consider the following ODE system:

$$
\dot{x}=f(x, \xi), \quad(x, \xi) \in \mathbb{R}^{4} \times \mathbb{R}^{l},
$$




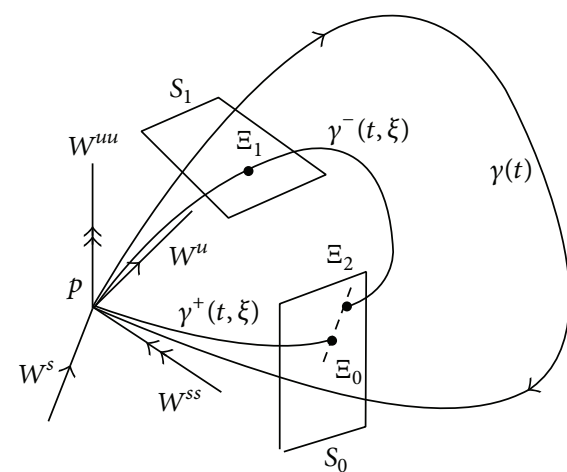

(a)

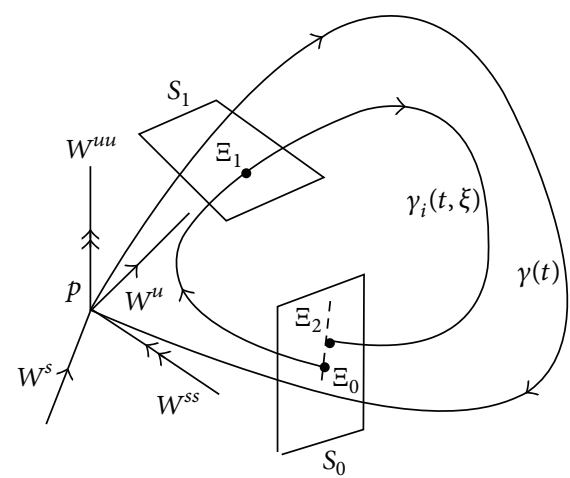

(b)

Figure 1: The gap $\left\|\gamma^{-}(T, \xi)-\gamma^{+}(T, \xi)\right\| \neq 0$ in (a); there is no homoclinic orbit in general. The gap $\left\|\gamma_{i}\left(T+T_{i}+\tau_{i}, \xi\right)-\gamma_{i}(T, \xi)\right\| \neq 0$ in $(\mathrm{b})$; there is no periodic orbit in general.

where $f$ is sufficiently smooth and $l \geq 4$. Suppose that the system (5) has an orbit $\gamma(t)$ of codimension-1 homoclinic to a saddle equilibrium $p$ at $\xi=0$. Let $T$ be a certain time, such that $\gamma(T)$ and $\gamma(-T)$ are in some small neighborhood $U$ of $p$. Then we can take two sections vertical to $T_{\gamma( \pm T)}$ :

$$
S_{0}:\{x \mid x=x(T)\} \subset U ; \quad S_{1}:\{x \mid x=x(-T)\} \subset U .
$$

Generally, if the small parameter $\xi \neq 0$, the homoclinic orbit $\gamma(t)$ will not exist. But the system (5) must have solutions $\gamma^{ \pm}(t, \xi)$ with the properties

$$
\begin{gathered}
\dot{\gamma}^{ \pm}=f\left(\gamma^{ \pm}, \xi\right), \\
\gamma^{+}(t, \xi) \in W^{s}(p), \quad \gamma^{-}(t, \xi) \in W^{u}(p), \\
\gamma^{ \pm}(t, 0)=\gamma(t), \\
\gamma^{+}(T, \xi) \in S_{0}, \quad \gamma^{-}(-T, \xi) \in S_{1}, \\
\left\|\gamma^{-}(T, \xi)-\gamma^{+}(T, \xi)\right\| \ll 1,
\end{gathered}
$$

where $W^{s}(p)$ and $W^{u}(p)$ are the stable and unstable manifolds of the equilibrium $p$, and $\operatorname{dim}\left(T_{\gamma(t)} W^{s} \cap T_{\gamma(t)} W^{u}\right)=1$. Notice that if the gap $\left\|\gamma^{-}(T, \xi)-\gamma^{+}(T, \xi)\right\|=0$ in the transverse section $S_{0}$, it means that the homoclinic orbit is kept (see Figure 1(a)) but it may not be of codimension-1.

Moreover, the system (5) still has other solutions $\gamma_{i}(t, \xi)$; $i$ is a natural number. Set the time of the orbit $\gamma_{i}(t, \xi)$ from $S_{0}$ to $S_{1}$ and from $S_{1}$ to $S_{0}$ to be $\tau_{i}$ and $T_{i}$, respectively; there are

$$
\begin{gathered}
\dot{\gamma}_{i}=f\left(\gamma_{i}, \xi\right), \\
\gamma_{i}\left(T+T_{i}+\tau_{i}, \xi\right), \quad \gamma_{i}(T, \xi) \in S_{0}, \\
\left\|\gamma_{i}\left(T+T_{i}+\tau_{i}, \xi\right)-\gamma_{i}(T, \xi)\right\| \ll 1 .
\end{gathered}
$$

Actually $\gamma_{i}(t, \xi)$ is a regular orbit and will be periodic if the gap $\left\|\gamma_{i}\left(T+T_{i}+\tau_{i}, \xi\right)-\gamma_{i}(T, \xi)\right\|=0$; namely, the orbit starting in $S_{0}$ will return to $S_{0}$ after the time $T_{i}+\tau_{i}$; see Figure 1(b).

From above, we see that the gap in the transverse section $S_{0}$ of some orbits is crucial to study bifurcations of the system. So in the next section we try to quantitate the gap size.

\section{Main Method}

To well carry out our discussion, we give some hypotheses for the system (5) here.

$\left(A_{1}\right)$ The spectrum $\sigma\left(D_{x} f(p, \xi)\right)=\left\{\lambda_{1}(\xi), \lambda_{2}(\xi),-\rho_{1}(\xi)\right.$, $\left.-\rho_{2}(\xi)\right\}$, and $\lambda_{2}(\xi)>\lambda_{1}(\xi)>0>-\rho_{1}(\xi)>-\rho_{2}(\xi)$.

$\left(A_{2}\right)$ As $t \rightarrow+\infty$, the homoclinic orbit $\gamma(t) \rightarrow p$ along the strong stable manifold $W^{s s}(p)$.

$\left(A_{3}\right)$ Vectors in the strong unstable (resp., stable) manifold $W^{u u}$ (resp., $W^{s s}$ ) return to the saddle $p$ in the direction along $W^{u}$ (resp., $W^{s}$ ).

We know that the discontinuity of the functions $\gamma^{ \pm}(t, \xi)$ or $\gamma_{i}(t, \xi)$ is confined in a special position in $S_{0}$. Since $\operatorname{dim}\left(T_{\gamma(t)} W^{s} \cap T_{\gamma(t)} W^{u}\right)=1$, the space

$$
\left(T_{\gamma(t)} W^{s} \cap T_{\gamma(t)} W^{u}\right)^{\perp}=\operatorname{span}\left\{\varphi_{1}\right\}
$$

is of one dimension, where $\varphi_{1}$ can be taken as the solution of the linear variational system

$$
\dot{y}=D_{x} f(\gamma(t), 0) y,
$$

and $\varphi_{1}(T)=\left(0, \omega_{12}, 1,0\right), \varphi_{1}(-T)=\left(\omega_{11}, 0, \omega_{13}, \omega_{14}\right)$ based on the assumptions of $\left(A_{2}\right)$ and $\left(A_{3}\right)$; refer to [13] for the details.

Beside this, by the theories of matrix, the other three solutions denoted by $\varphi_{2}, \varphi_{3}$, and $\varphi_{4}$ of the system (10) can also be taken in the following ways:

$$
\begin{gathered}
\varphi_{2}=\frac{-\dot{\gamma}(t)}{|\dot{\gamma}(T)|} \in T_{\gamma(t)} W^{u} \cap T_{\gamma(t)} W^{s}, \\
\varphi_{3} \in T_{\gamma(t)} W^{u}, \quad \varphi_{4} \in T_{\gamma(t)} W^{s},
\end{gathered}
$$

satisfying

$$
\begin{gathered}
\varphi_{2}(-T)=\left(\omega_{21}, 0,0,0\right), \quad \varphi_{2}(T)=(0,0,0,1), \\
\varphi_{3}(-T)=(0,0,1,0), \quad \varphi_{3}(T)=\left(\omega_{31}, \omega_{32}, 0, \omega_{34}\right), \\
\varphi_{4}(-T)=\left(\omega_{41}, \omega_{42}, \omega_{43}, \omega_{44}\right), \quad \varphi_{4}(T)=(0,1,0,0) .
\end{gathered}
$$


Now take a transformation

$$
\begin{aligned}
x(t)= & \gamma(t)+\Phi(t) \Xi=\gamma(t)+\varphi_{1}(t) \chi_{1} \\
& +\varphi_{3}(t) \chi_{3}+\varphi_{4}(t) \chi_{4},
\end{aligned}
$$

where $\Phi(t)=\left(\varphi_{1}(t), \varphi_{2}(t), \varphi_{3}(t), \varphi_{4}(t)\right)$ and $\Xi=\left(\chi_{1}, 0\right.$, $\left.\chi_{3}, \chi_{4}\right)^{*}$. Then system (5) becomes the following ODE in the new variable $\Xi$; namely,

$$
\begin{aligned}
\dot{\Xi}= & \Phi^{-1} D_{\xi} f(\gamma(t), 0) \xi+\Phi^{-1} D_{x \xi}^{2} f(\gamma(t), 0) \Phi \Xi \xi \\
& +O\left(|\Phi||\Xi|^{2}\right)+O\left(|\Phi|^{-1}|\xi|^{2}\right) .
\end{aligned}
$$

By (14),

$$
\int_{-T}^{T} \dot{\Xi} \mathrm{d} t=\int_{-T}^{T} \Phi^{-1} D_{\xi} f(\gamma(t), 0) \xi \mathrm{d} t+\text { h.o.t. }
$$

gives

$$
\Xi(T)=\Xi(-T)+M \xi+\text { h.o.t., }
$$

where $M=\left(M_{1}, 0, M_{3}, M_{4}\right)^{*}=\int_{-T}^{T} \Phi^{-1} D_{\xi} f(\gamma(t), 0) \mathrm{d} t$.

Notice that in (13), $\Xi$ represents in some meaning the deviation in the normal direction of the manifolds $T_{\gamma(t)} W^{s} \cap$ $T_{\gamma(t)} W^{u}$, so $\Xi(-T) \in S_{1}$ and $\Xi(T) \in S_{0}$; in other words, (16) maps a point in $S_{1}$ to a point in $S_{0}$.

On the other hand, from assumption $\left(A_{1}\right)$, system $(5)$ admits a local linearization

$$
\begin{aligned}
D_{x} f(p, \xi)= & \lambda_{1}(\xi) x_{1} \frac{\partial}{\partial x_{1}}-\rho_{1}(\xi) x_{2} \frac{\partial}{\partial x_{2}} \\
& +\lambda_{2}(\xi) x_{3} \frac{\partial}{\partial x_{3}}-\rho_{2}(\xi) x_{4} \frac{\partial}{\partial x_{4}},
\end{aligned}
$$

where $x=\left(x_{1}, x_{2}, x_{3}, x_{4}\right)$.

Suppose $\Xi(T)=\Xi_{0}=\left(x_{1}^{0}, x_{2}^{0}, x_{3}^{0}, x_{4}^{0}\right)$ and $\Xi(-T)=\Xi_{1}=$ $\left(x_{1}^{1}, x_{2}^{1}, x_{3}^{1}, x_{4}^{1}\right)$. Rescale the time $s=e^{-\lambda_{1}(\xi) \tau}$ and write $\alpha=$ $\rho_{1}(\xi) / \lambda_{1}(\xi), \beta=\rho_{2}(\xi) / \lambda_{1}(\xi)$, and $\nu=\lambda_{2}(\xi) / \lambda_{1}(\xi)$. Without loss of generality, we assume $\alpha \geq 1$ for sufficiently small $\xi$. Otherwise, we can set $s=e^{-\rho_{1}(\xi) \tau}$ and $\alpha=\lambda_{1}(\xi) / \rho_{1}(\xi)$. Clearly, $\beta>\alpha \geq 1$.

Then by the linear approximation solutions of system (17), we have thereby

$$
\begin{aligned}
x= & e^{\lambda_{1}(\xi) \tau} x_{1}^{0} \frac{\partial x}{\partial x_{1}}+e^{-\rho_{1}(\xi) \tau} x_{2}^{0} \frac{\partial x}{\partial x_{2}}+e^{\lambda_{2}(\xi) \tau} x_{3}^{0} \frac{\partial x}{\partial x_{3}} \\
& +e^{-\rho_{2}(\xi) \tau} x_{4}^{0} \frac{\partial x}{\partial x_{4}} \\
= & s^{-1} x_{1}^{0} \frac{\partial x}{\partial x_{1}}+s^{\alpha} x_{2}^{0} \frac{\partial x}{\partial x_{2}}+s^{-v} x_{3}^{0} \frac{\partial x}{\partial x_{3}}+s^{\beta} x_{4}^{0} \frac{\partial x}{\partial x_{4}} .
\end{aligned}
$$

Formula (18) indeed maps a point in $S_{0}$ to a point in $S_{1}$ in some subset of $U$ if we substitute $x$ by $\Xi_{1}$.

Now take $\Xi_{0} \in S_{0}$ as the initial point. System (5) must have an orbit $\gamma(t, \xi)$ starting at $\Xi_{0}$, passing through $S_{1}$ with an intersection $\Xi_{1}$, and finally returning to $S_{0}$ at some point $\Xi_{2}$; see Figure 1. From (13), (16), and (18), we can derive $\Xi_{2}-\Xi_{0}=$ $\left(\chi_{1}^{2}, 0, \chi_{3}^{2}, \chi_{4}^{2}\right)-\left(\chi_{1}^{0}, 0, \chi_{3}^{0}, \chi_{4}^{0}\right) \triangleq\left(\varepsilon_{1}(s, \xi), 0, \varepsilon_{3}(s, \xi), \varepsilon_{4}(s, \xi)\right)$, where

$$
\begin{aligned}
\omega_{14} \varepsilon_{1}(s, \xi)= & \delta s^{\beta}-\omega_{14} x_{3}^{1} s^{\nu}-\omega_{44} \omega_{42}^{-1} s^{\alpha} x_{2}^{0} \\
& +\omega_{14} M_{1} \xi+\text { h.o.t., } \\
\varepsilon_{3}(s, \xi)= & x_{3}^{1}-\omega_{31}^{-1} \delta s+\left(\omega_{13} \omega_{44} \omega_{14}^{-1}-\omega_{43}\right) \omega_{42}^{-1} s^{\alpha} x_{2}^{0} \\
& +M_{3} \xi+\text { h.o.t., } \\
\varepsilon_{4}(s, \xi)= & \omega_{42}^{-1} s^{\alpha} x_{2}^{0}-x_{2}^{0}+\omega_{12} s^{\nu} x_{3}^{1}+\omega_{32} \omega_{31}^{-1} \delta s \\
& +M_{4} \xi+\text { h.o.t. }
\end{aligned}
$$

Denote $a=\omega_{44} w_{42}^{-1}, b=\omega_{32} w_{31}^{-1}$, and $\omega=\omega_{14}$. Then the gap between the points $\Xi_{2}$ and $\Xi_{0}$ can be represented by (refer to [13-15])

$$
\begin{aligned}
\gamma(2 T+\tau, \xi)-\gamma(T, \xi) \\
=\varepsilon_{\gamma}(s, \xi) \\
=-a M_{4} \xi s^{\alpha}+\delta s^{\beta}+\omega M_{3} \xi s^{\nu}-a b \delta s^{1+\alpha} \\
\quad+\omega M_{1} \xi+O\left(s^{1+\nu}\right) .
\end{aligned}
$$

Obviously, $\varepsilon_{\gamma}(s, \xi)=0$ means that system (5) has closed orbits.

\section{Saddle-Node Bifurcations}

From this section, we analyze bifurcation construction of system (5). Firstly, set $r=s^{\alpha}$. Then $\varepsilon_{\gamma}(r, \xi)=0$ equals

$$
\begin{aligned}
& a M_{4} \xi r-\delta r^{\beta / \alpha}-\omega M_{3} \xi r^{\nu / \alpha}+a b \delta r^{1+(1 / \alpha)}-\omega M_{1} \xi \\
& \quad+O\left(r^{(1+\nu) / \alpha}\right)=0
\end{aligned}
$$

or

$$
\begin{aligned}
r= & \frac{\delta}{a M_{4} \xi} r^{\beta / \alpha}+\frac{\omega M_{3} \xi}{a M_{4} \xi} r^{\nu / \alpha}-\frac{a b \delta}{a M_{4} \xi} r^{1+(1 / \alpha)} \\
& +\frac{\omega M_{1} \xi}{a M_{4} \xi}+\frac{1}{a M_{4} \xi} O\left(r^{(1+\nu) / \alpha}\right) .
\end{aligned}
$$

Define $S(\xi)=\omega M_{1} \xi / a M_{4} \xi$. When $\|S(\xi)\|=$ $O\left(\left\|M_{4} \xi\right\|^{\alpha /(\beta-\alpha)}\right), a M_{4} \xi r$ is the leading term in (21), so (21) has a small solution $r=S(\xi)+$ h.o.t. $>0$; but as $\|S(\xi)\| \gg\left\|M_{4} \xi\right\|^{\alpha /(\beta-\alpha)}, r=\left(-\delta^{-1} \omega M_{1} \xi\right)^{\alpha / \beta}+$ h.o.t. > 0. No matter which case, a periodic orbit of system (5) exists.

Theorem 1. Under $\left(A_{1}\right)-\left(A_{3}\right)$ and for $1+\alpha>\beta$, system (5) has a 1-periodic orbit.

To look for saddle-node bifurcations of 1-periodic orbits, it is enough to differentiate (22) with respect to $r$. Consider

$$
1=\frac{\beta \delta}{\alpha a M_{4} \xi} r^{(\beta / \alpha)-1}+\frac{\nu \omega M_{3} \xi}{\alpha a M_{4} \xi} r^{(\nu / \alpha)-1}+\frac{1}{a M_{4} \xi} O\left(r^{1 / \alpha}\right) .
$$


Solving (23) for $r$, there is

$$
\begin{aligned}
r= & \left(\frac{\alpha a M_{4} \xi}{\beta \delta}\right)^{\alpha /(\beta-\alpha)} \\
& +O\left(\left\|M_{3} \xi\right\|^{\alpha /(\beta-\alpha)}\left\|M_{4} \xi\right\|^{\alpha(\nu-\alpha) /(\beta-\alpha)^{2}}\right) .
\end{aligned}
$$

Then substituting (24) into (22), an asymptotic expression for a saddle-node bifurcation is given by

$$
\begin{aligned}
S(\xi)= & \frac{\beta-\alpha}{\beta}\left(\frac{\alpha a M_{4} \xi}{\beta \delta}\right)^{\alpha /(\beta-\alpha)} \\
& +O\left(\left\|M_{3} \xi\right\|\left\|M_{4} \xi\right\|^{(\nu-\beta+\alpha) /(\beta-\alpha)}\right) .
\end{aligned}
$$

Furthermore, if we continue to differentiate (23), there is

$$
\begin{aligned}
0= & \frac{(\beta-\alpha) \beta \delta}{\alpha^{2} a M_{4} \xi} r^{(\beta / \alpha)-2}+\frac{(\nu-\alpha) \nu \omega M_{3} \xi}{\alpha^{2} a M_{4} \xi} r^{(\nu / \alpha)-2} \\
& +\frac{1}{a M_{4} \xi} O\left(r^{(1-\alpha) / \alpha}\right) .
\end{aligned}
$$

Equation (26) is solvable for $\beta>v$ with

$$
r=\left(-\frac{(\nu-\alpha) \nu \omega M_{3} \xi}{(\beta-\alpha) \delta \beta}\right)^{\alpha /(\beta-\gamma)}+O\left(\left\|M_{3} \xi\right\|^{\alpha(1-\alpha) /(\beta-\nu)^{2}}\right)
$$

This is a triple solution of (22). It means that a saddle-node bifurcation of a triple 1-periodic orbit exists. The asymptotic expression can be derived from (22) and (23):

$$
S(\xi)=\left(-\frac{(\nu-\alpha) \nu \omega M_{3} \xi}{(\beta-\alpha) \delta \beta}\right)^{\alpha /(\beta-\nu)}+O\left(\left\|M_{3} \xi\right\|^{\alpha /(\beta-\nu)}\right),
$$

where

$$
\begin{aligned}
\omega M_{3} \xi= & -\frac{(\beta-\alpha) \delta \beta}{(\nu-\alpha) \nu}\left(\frac{\alpha(\nu-\alpha) a M_{4} \xi}{\delta \beta(\nu-\beta)}\right)^{(\beta-\nu) /(\beta-\alpha)} \\
& +o\left(\left\|M_{4} \xi\right\|^{(\beta-\nu) /(\beta-\alpha)}\right)
\end{aligned}
$$

Theorem 2. Under $\left(A_{1}\right)-\left(A_{3}\right)$ and for $1+\alpha>\beta$, system (5) has a saddle-node bifurcation SN of a double 1-periodic orbit given by (25) in the parameter space; moreover, for $\beta>v$, system (5) has a saddle-node bifurcation $S N^{2}$ of a triple 1periodic orbit given by (28).

Remark 3. For the case $\beta<v,(26)$ has no sufficiently small positive solution, so there does not exist $n$-multiple 1-periodic orbit bifurcation for $n \geq 3$.

Now we define a surface in the parameter space of $\xi$ :

$$
H_{1}(\xi)=\left\{\xi: M_{1} \xi+o(1)=0\right\} .
$$

On the surface $H_{1}$, (21) equals

$$
\begin{aligned}
& r\left(a M_{4} \xi-\delta r^{(\beta / \alpha)-1}-\omega M_{3} \xi r^{(\nu / \alpha)-1}+a b \delta r^{1 / \alpha}\right. \\
& \left.+O\left(r^{((1+\nu) / \alpha)-1}\right)\right)=0 .
\end{aligned}
$$

Clearly, it has a zero solution $r_{1}=0$. If we differentiate the part in the parentheses in (31) for $r$, we get

$$
-\frac{\beta-\alpha}{\alpha} \delta r^{(\beta / \alpha)-2}-\frac{\nu-\alpha}{\alpha} \omega M_{3} \xi r^{(\nu / \alpha)-2}+O\left(r^{(1 / \alpha)-1}\right)=0 .
$$

It has a solution for $\beta>v$ :

$$
r_{2}=\left(-\frac{\nu-\alpha}{(\beta-\alpha) \delta} \omega M_{3} \xi\right)^{\alpha /(\beta-v)}+O\left(\left\|M_{3} \xi\right\|^{\alpha(1+\alpha-\nu) /(\beta-\gamma)^{2}}\right) .
$$

Then we obtain another saddle-node bifurcation similarly:

$$
\begin{aligned}
R(\xi)= & \frac{\beta-\nu}{\beta-\alpha}\left(-\frac{\nu-\alpha}{(\beta-\alpha) \delta} \omega M_{3} \xi\right)^{(\nu-\alpha) /(\beta-\nu)} \\
& +O\left(\left\|M_{3} \xi\right\|^{(1+\nu-\beta) /(\beta-\nu)}\right)
\end{aligned}
$$

where $R(\xi)=a M_{4} \xi / \omega M_{3} \xi$.

Notice that (32) has no solution for $\beta<\nu$. But from (31), a small positive solution in the form $r_{2}^{\prime}=\left(\delta^{-1} a M_{4} \xi\right)^{\alpha /(\beta-\alpha)}+$ $\mathrm{O}\left(\left\|M_{3} \xi\right\|^{\alpha /(\beta-\alpha)}\left\|M_{4} \xi\right\|^{\alpha(\nu-\alpha) /(\beta-\alpha)^{2}}\right)$ exists.

So we can conclude the following.

Theorem 4. Under $\left(A_{1}\right)-\left(A_{3}\right)$ and for $1+\alpha>\beta>\nu$, system (5) has a homoclinic-saddle-node bifurcation HSN of a 1-homoclinic orbit and a double 1-periodic orbit confined on $H_{1} \cap R(\xi)$ while, for $\beta<v$, system (5) has only a 1-homoclinic orbit and a 1-periodic orbit in the parameter space and the 1homoclinic orbit is of codimension-1.

Remark 5. In Theorem 4, the 1-homoclinic orbit may be nongeneral, that is, may be a flip orbit, because the orbit can connect the saddle along the weak unstable and strong stable directions if $M_{4} \xi=0$.

\section{Homoclinic-Doubling and Periodic-Doubling Bifurcations}

Now we focus on 2-homoclinic orbits and 2-periodic orbits. Correspondingly, the gap functions are

$$
\begin{aligned}
& r_{1}-\frac{\delta}{a M_{4} \xi} r_{1}^{\beta / \alpha}-\frac{\omega M_{3} \xi}{a M_{4} \xi} r_{2}^{\nu / \alpha}+\frac{a b \delta}{a M_{4} \xi} r_{1}^{1+(1 / \alpha)}-S(\xi) \\
& +\frac{1}{a M_{4} \xi} O\left(r_{1}^{1 / \alpha} r_{2}^{\nu / \alpha}\right)=0, \\
& r_{2}-\frac{\delta}{a M_{4} \xi} r_{2}^{\beta / \alpha}-\frac{\omega M_{3} \xi}{a M_{4} \xi} r_{1}^{\nu / \alpha}+\frac{a b \delta}{a M_{4} \xi} r_{2}^{1+(1 / \alpha)}-S(\xi) \\
& +\frac{1}{a M_{4} \xi} O\left(r_{2}^{1 / \alpha} r_{1}^{\nu / \alpha}\right)=0 .
\end{aligned}
$$




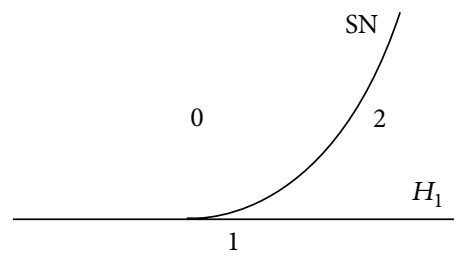

(a)

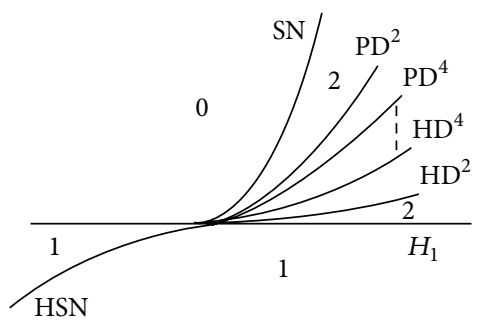

(b)

FIGURE 2: Bifurcation surfaces for $1+\alpha>\beta, v>\beta$ in (a) and for $1+\alpha>\beta>v$ in (b). 0 means no periodic orbits and $n$ means $n$ periodic orbits. Chaos occurs in the region bounded by $\mathrm{HD}^{2^{n}}$ and $\mathrm{PD}^{2^{n}}$.

To find a 2-homoclinic orbit, the above two equations must have a kind of solutions with $r_{1}=0$ and $r_{2}>0$. That is,

$$
\begin{gathered}
r_{2}^{\nu / \alpha}+\frac{\omega M_{1} \xi}{\omega M_{3} \xi}+\frac{1}{\omega M_{3} \xi} O\left(r_{2}^{(\nu+\beta) / \alpha}\right)=0, \\
r_{2}-\frac{\delta}{a M_{4} \xi} r_{2}^{\beta / \alpha}+\frac{a b \delta}{a M_{4} \xi} r_{2}^{1+(1 / \alpha)}-S(\xi) \\
+\frac{1}{a M_{4} \xi} O\left(r_{2}^{(1+\beta) / \alpha}\right)=0 .
\end{gathered}
$$

Then we get

$$
\begin{aligned}
S(\xi)= & \left(-\frac{M_{1} \xi}{M_{3} \xi}\right)^{\alpha / \nu}-\frac{\delta}{a M_{4} \xi}\left(-\frac{M_{1} \xi}{M_{3} \xi}\right)^{\beta / \nu} \\
& +\frac{1}{a M_{4} \xi} O\left(\left\|\frac{M_{1} \xi}{M_{3} \xi}\right\|^{(1+\alpha) / v}\right),
\end{aligned}
$$

in the region defined by $\left\|M_{1} \xi\right\| \ll\left\|M_{3} \xi\right\|^{\beta /(\beta-\nu)}$

To find a 2-periodic orbit, the gap functions will have two positive solutions $r_{1}$ and $r_{2}$. We suppose that $r_{2}=(1+\epsilon) r_{1}$ after the reparametrization $\xi=\left(\xi_{1}, \epsilon\right)$. Then there are

$$
\begin{aligned}
& r_{1}-\frac{\delta}{a M_{4} \xi} r_{1}^{\beta / \alpha}-\frac{\omega M_{3} \xi}{a M_{4} \xi}(1+\epsilon)^{\nu / \alpha} r_{1}^{\nu / \alpha}-S(\xi) \\
& \quad+\frac{1}{a M_{4} \xi} O\left(r_{1}^{(1+\alpha) / \alpha}\right)=0, \\
& (1+\epsilon) r_{1}-\frac{\delta}{a M_{4} \xi}(1+\epsilon)^{\beta / \alpha} r_{1}^{\beta / \alpha}-\frac{\omega M_{3} \xi}{a M_{4} \xi} r_{1}^{\nu / \alpha} \\
& -S(\xi)+\frac{1}{a M_{4} \xi} O\left((1+\epsilon)^{(1+\alpha) / \alpha} r_{1}^{(1+\alpha) / \alpha}\right)=0 .
\end{aligned}
$$

Subtracting the two equations, there is

$$
\begin{aligned}
r_{1}= & \left(\frac{\delta^{-1} \epsilon}{(1+\epsilon)^{\beta / \alpha}-1}\right)^{\alpha /(\beta-\alpha)}\left(a M_{4} \xi\right)^{\alpha /(\beta-\alpha)} \\
& +O\left(\left\|M_{3} \xi\right\|^{\alpha /(\beta-\alpha)}\|\epsilon\| r_{1}^{(\nu-\alpha) /(\beta-\alpha)}\right) .
\end{aligned}
$$

Finally, we get the 2-periodic orbit bifurcation:

$$
\begin{aligned}
S(\xi)= & \left(1-\frac{\epsilon}{(1+\epsilon)^{\beta / \alpha}-1}\right)\left(\frac{\delta^{-1} \epsilon}{(1+\epsilon)^{\beta / \alpha}-1}\right)^{\alpha /(\beta-\alpha)} \\
& \times\left(a M_{4} \xi\right)^{\alpha /(\beta-\alpha)}+O\left(\left\|M_{3} \xi\right\|\left\|M_{4} \xi\right\|^{(\alpha+\nu-\beta) /(\beta-\alpha)}\right) .
\end{aligned}
$$

Remark 6. Obviously, if $r_{1}=r_{2}$, the 2 -periodic orbit is close to the double 1-periodic orbit perturbed from the saddle-node bifurcation. This is true by taking limit $\epsilon \rightarrow 0$ in (40), and one may get the similar approximate expression given in (25).

If we continue the computation, we can finally get an asymptotic expression of the homoclinic-doubling bifurcation of $2^{n}$-homoclinic orbit and the periodic-doubling bifurcation of $2^{n}$-periodic orbit with the same leading terms as in (37) and (40), respectively. For example, for a 4-homoclinic orbit or a 4-periodic orbit, the gap functions are

$$
\begin{aligned}
& r_{1}-\frac{\delta}{a M_{4} \xi} r_{1}^{\beta / \alpha}-\frac{\omega M_{3} \xi}{a M_{4} \xi} r_{2}^{\nu / \alpha}+\frac{a b \delta}{a M_{4} \xi} r_{1}^{1+(1 / \alpha)} \\
& -S(\xi)+\frac{1}{a M_{4} \xi} o(1)=0, \\
& r_{2}-\frac{\delta}{a M_{4} \xi} r_{2}^{\beta / \alpha}-\frac{\omega M_{3} \xi}{a M_{4} \xi} r_{3}^{\nu / \alpha}+\frac{a b \delta}{a M_{4} \xi} r_{2}^{1+(1 / \alpha)} \\
& -S(\xi)+\frac{1}{a M_{4} \xi} o(1)=0, \\
& r_{3}-\frac{\delta}{a M_{4} \xi} r_{3}^{\beta / \alpha}-\frac{\omega M_{3} \xi}{a M_{4} \xi} r_{4}^{\nu / \alpha}+\frac{a b \delta}{a M_{4} \xi} r_{3}^{1+(1 / \alpha)} \\
& -S(\xi)+\frac{1}{a M_{4} \xi} o(1)=0, \\
& r_{4}-\frac{\delta}{a M_{4} \xi} r_{4}^{\beta / \alpha}-\frac{\omega M_{3} \xi}{a M_{4} \xi} r_{1}^{\nu / \alpha}+\frac{a b \delta}{a M_{4} \xi} r_{4}^{1+(1 / \alpha)} \\
& -S(\xi)+\frac{1}{a M_{4} \xi} o(1)=0 .
\end{aligned}
$$

We need only to consider solutions $r_{1}=0$ and $r_{i}>0, i=$ $2,3,4$, for the 4-homoclinic orbit or all the positive solutions for 4-periodic orbit. For concision, we omit the details here.

Now we can claim our last theorem. 
Theorem 7. Under $\left(A_{1}\right)-\left(A_{3}\right)$ and for $1+\alpha>\beta>v$, system (5) has a homoclinic-doubling bifurcation $\mathrm{HD}^{2^{n}}$ of $2^{n}$ homoclinic orbit and a periodic-doubling bifurcation $P D^{2^{n}}$ of $2^{n}$-periodic orbit defined by (37) and (40), respectively, in the parameter region $\left\|M_{1} \xi\right\| \ll\left\|M_{3} \xi\right\|^{\beta /(\beta-\nu)}$.

From the above analysis, one may see that all of these bifurcation surfaces have the same order $S(\xi)=$ $O\left(\left\|M_{4} \xi\right\|^{\alpha /(\beta-\alpha)}\right)$ except HSN and are tangent to $H_{1}$. To be clear, we illustrate these bifurcation surfaces in the parameter plane in Figure 2.

\section{Conflict of Interests}

The authors declare that there is no conflict of interests regarding the publication of this paper.

\section{Acknowledgments}

The authors would like to acknowledge the support from the National Natural Science Foundation of China (11271260) and the Innovation Program of Shanghai Municipal Education Commission (13ZZ116).

\section{References}

[1] M. Krupa, B. Sandstede, and P. Szmolyan, "Fast and slow waves in the FitzHugh-Nagumo equation," Journal of Differential Equations, vol. 133, no. 1, pp. 49-97, 1997.

[2] A. R. Champneys and M. D. Groves, "A global investigation of solitary-wave solutions to a two-parameter model for water waves," Journal of Fluid Mechanics, vol. 342, pp. 199-229, 1997.

[3] A. J. Homburg, H. Kokubu, and V. Naudot, "Homoclinic-doubling cascades," Archive for Rational Mechanics and Analysis, vol. 160, no. 3, pp. 195-243, 2001.

[4] M. Kisaka, H. Kokubu, and H. Oka, "Supplement to homoclinic-doubling bifurcation in vector fields," in Dynamical Systems, pp. 92-116, Longman, Harlow, UK, 1993.

[5] H. Kokubu, M. Komuro, and H. Oka, "Multiple homoclinic bifurcations from orbit-flip. I. Successive homoclinic doublings," International Journal of Bifurcation and Chaos in Applied Sciences and Engineering, vol. 6, no. 5, pp. 833-850, 1996.

[6] C. A. Morales and M. J. Pacifico, "Inclination-flip homoclinic orbits arising from orbit-flip," Nonlinearity, vol. 14, no. 2, pp. 379-393, 2001.

[7] B. Sandstede, Verzweigungstheorie Homokliner Verdopplungen [Ph.D. thesis], Institut fur Angewandte Analysis und Stochastic, Freie Universitat Berlin, Berlin, Germany, 1993.

[8] A. J. Homburg and B. Krauskopf, "Resonant homoclinic flip bifurcations," Journal of Dynamics and Differential Equations, vol. 12, no. 4, pp. 807-850, 2000.

[9] B. E. Oldeman, B. Krauskopf, and A. R. Champneys, "Numerical unfoldings of codimension-three resonant homoclinic flip bifurcations," Nonlinearity, vol. 14, no. 3, pp. 597-621, 2001.

[10] Q. Lu, Z. Qiao, T. Zhang, and D. Zhu, "Heterodimensional cycle bifurcation with orbit-flip," International Journal of Bifurcation and Chaos in Applied Sciences and Engineering, vol. 20, no. 2, pp. 491-508, 2010.
[11] X. Liu, "Homoclinic flip bifurcations accompanied by transcritical bifurcation," Chinese Annals of Mathematics B, vol. 32, no. 6, pp. 905-916, 2011.

[12] Y. Xu, D. Zhu, and X. Liu, "Bifurcations of multiple homoclinics in general dynamical systems," Discrete and Continuous Dynamical Systems A, vol. 30, no. 3, pp. 945-963, 2011.

[13] T. Zhang and D. Zhu, "Bifurcations of homoclinic orbit connecting two nonleading eigendirections," International Journal of Bifurcation and Chaos in Applied Sciences and Engineering, vol. 17, no. 3, pp. 823-836, 2007.

[14] Q. Tian and D. Zhu, "Bifurcations of nontwisted heteroclinic loop," Science in China A, vol. 43, no. 8, pp. 818-828, 2000.

[15] D. M. Zhu and Z. H. Xia, "Bifurcations of heteroclinic loops," Science in China A, vol. 41, no. 8, pp. 837-848, 1998. 


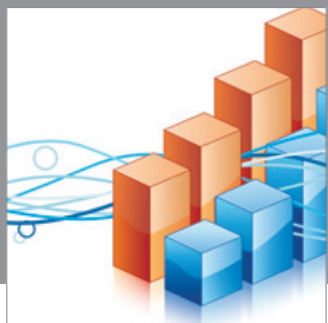

Advances in

Operations Research

mansans

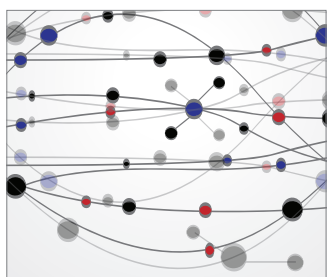

The Scientific World Journal
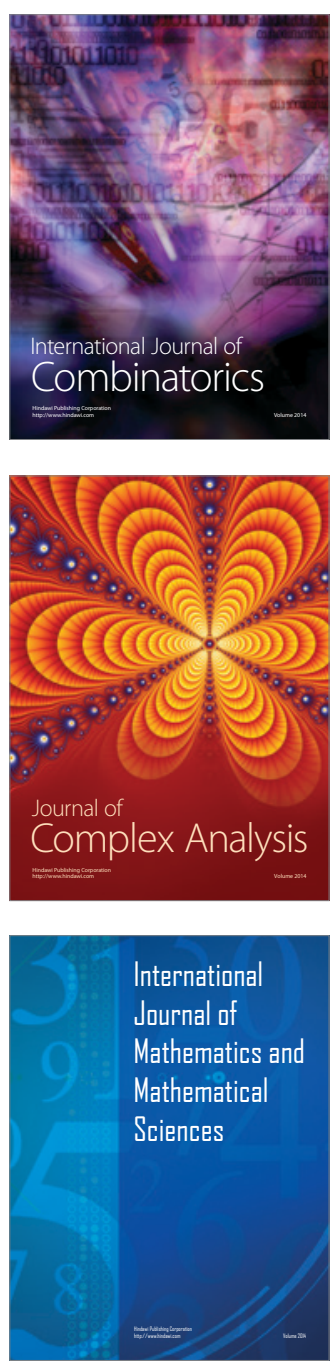
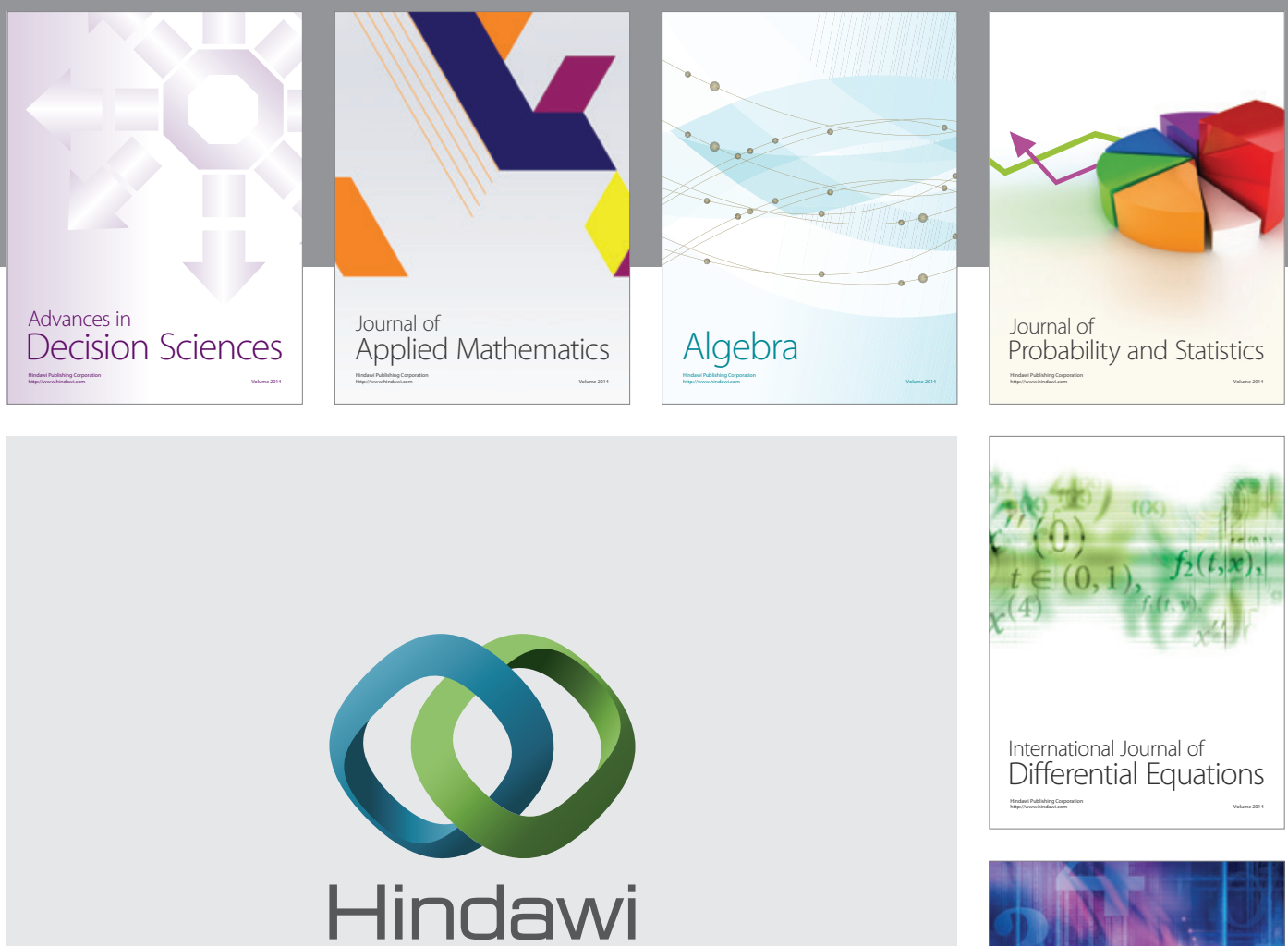

Submit your manuscripts at http://www.hindawi.com
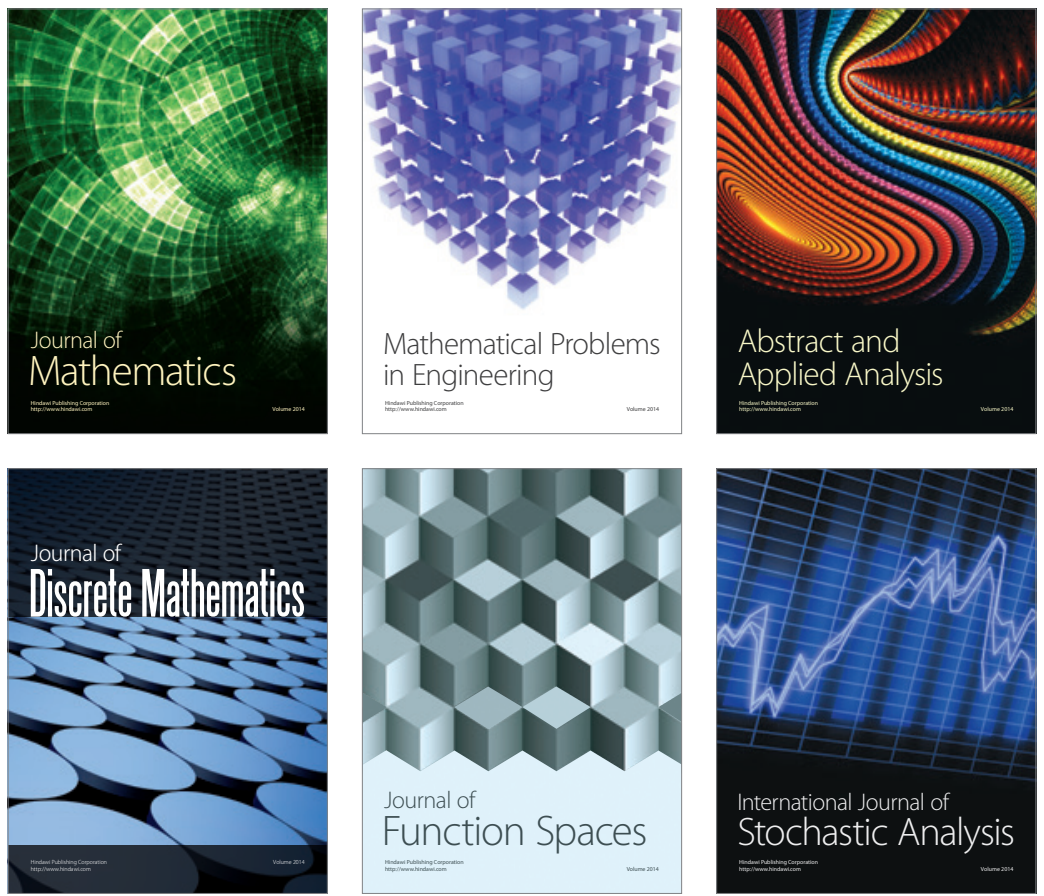

Journal of

Function Spaces

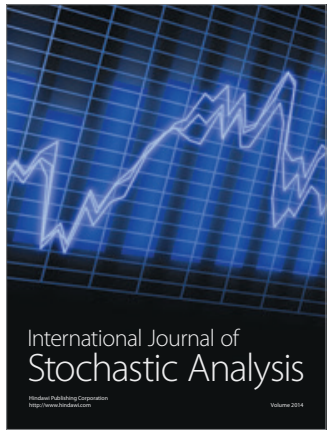

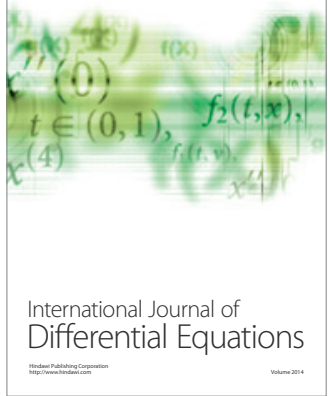
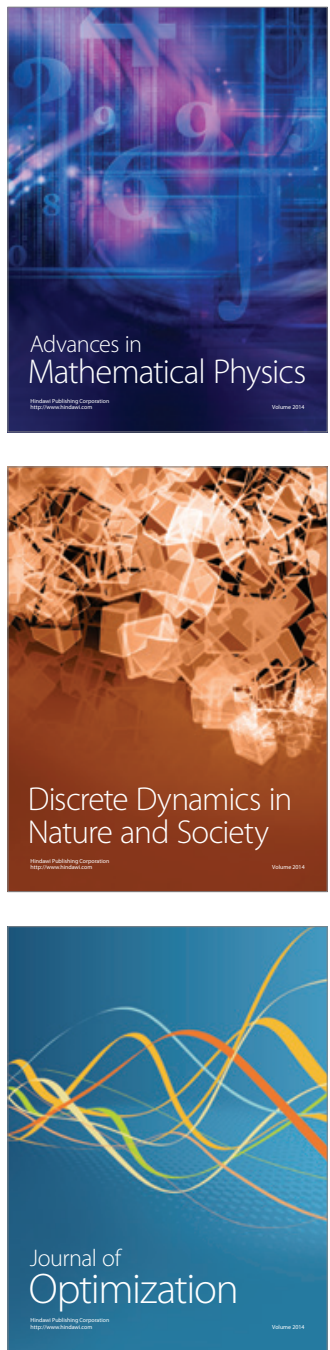